\title{
Ripple's Market Competitiveness in the Long-term
}

\author{
Shanshan Chen ${ }^{1, \mathrm{a}, \uparrow}$, Ninghui Wang ${ }^{2, *}, \mathrm{~b}, \uparrow$, Yuxin Zhong ${ }^{3, *}, \mathrm{c}, \uparrow$ \\ ${ }^{1}$ College of Software and Microelectronics, Peking University, Beijing, 100871, China \\ ${ }^{2}$ Department of Economics, University of California Santa Barbara, Santa Barbara, 93106, United States \\ ${ }^{3}$ Department of Statistics, University of Michigan Ann Arbor, Michigan, 48105, United States \\ "Corresponding author.Email: ${ }^{a} 1055289540 @ q q . c o m,{ }^{b}$ ninghuiwang@ucsb.edu, ${ }^{c}$ zhongyx@umich.edu \\ These authors contributed equally.
}

\begin{abstract}
With Ripple becoming the first cross-border payment system open to global financial institutions globally, faster, safer, and cheaper cross-border payment by bypassing expensive intermediaries has been realized. This paper aims to explain how Ripple's long-term competitiveness can make such an efficient ecosystem and promote the development of global payment. This paper demonstrates Ripple's long-term competitiveness from three perspectives. Firstly, we compared the currency Ripple (XRP) with other cryptocurrencies in terms of future value-added, remittance fees, and currency exchange methods and show that XPR's historical value is relatively stable and its future expectation is promising. Secondly, from the basic principles and characteristics of RippleNet operation, this paper discusses the security and privacy protection advantages of the RippleNet system. Finally, from the perspective of the company Ripple Labs, this paper analyzes the supporting forces behind Ripple, which will help us better understand how Ripple stands out from other cryptocurrencies. This paper suggests that based on its cross-border payment services, Ripple has a strong competitive advantage over its peers either as a cryptocurrency and a cross-border payment tool (vehicle). This study also provides a reference for Ripple to demonstrate that it has a large market space and long-term competitiveness in cross-border payment, and virtual currency investment has entered a new frontier.
\end{abstract}

Keywords: Cross-border payment, RippleNet, Cryptocurrency.

\section{INTRODUCTION}

With the development of cryptocurrency nowadays, more and more cryptocurrencies appear in the market: Bitcoin, Ethereum, XRP, etc. As an outstanding example in the cryptocurrency market, XRP has shown its strong competitiveness in the past. As an efficient tool for cross-border transactions, the whole Ripple system means a lot more than just a type of cryptocurrency. In this paper, we aim to evaluate the XRP's market competitiveness in the long term and examine its correlation with the cryptocurrency market.

Existing research on Ripple's advantages is mostly based on comparison with other currencies or virtual currency markets and cross-border payments. Deng took Ripple as an example to discuss the application of cross-border payment under blockchain technology and summarized two representative operation modes of blockchain [1]. Shao concluded the fundamental structure of blockchain technology, which generally includes the data layer, network layer, intelligent contract layer, consensus layer, and application layer
[2]. Jiang analyzed the advantages and disadvantages of several existing cross-border payment methods and found that the most common cross-border payment mode is SWIFT wire transfer. Youssef discussed Ripple's security and privacy provisions on the Bitcoin system and found that the current choice of parameters does not prevent the occurrence of forks in the system [3]. Although cryptocurrency has received considerable attention in previous studies, most of these are studies on the virtual currency market or comparing XRP with other cryptocurrencies, without in-depth research on Ripple's long-term core competitiveness. This article fills this gap by analyzing its price stability and market expectations in the entire market.

The first part evaluates the basic element of every cryptocurrency: the value in the whole market. XRP was always known for its low and stable price, while we know the price of most cryptocurrencies is always fluctuating. Although a fairly stable price sometimes means a bad one for investment, it's still worth discussing that could be a superiority from another 
perspective. Could XRP be considered as a potential asset, or could the value get rising in the future? This passage will analyze XRP's price in the whole market when comparing it with some other cryptocurrencies and give some reasonable predictions in relation to its future change.

The second part outlines Ripple's operating system and evaluates the network that supports the operation of Ripple: RippleNet. Many cryptocurrencies use blockchain technology, which is naturally related to a decentralized network. While Ripple shows its controversy because it has blockchain technology and relatively centralized characteristics simultaneously, it's our target to explore whether these two concepts really contradict each other. How does XRP survive as a special cryptocurrency that owns two characteristics at the same time, and does this stimulate Ripple's development to some degree? With our further understanding of RippleNet, we'll also focus on one of the necessary questions for digital currency: security and privacy protection. Only the recognition of the Ripple system's safety can ensure its future opportunity.

The third part talks about the power behind Ripple, the Ripple Labs Inc. that maintains Ripple's competitiveness. The XRP and RippleNet we mentioned will not exist without the effort of Ripple Labs, and it's this company that contributes so much to Ripple's valuation and improvement. We will introduce Ripple Labs's background, including its operating model and current situation. This shall also help us understand the relationship between Ripple and the company. Besides, we will also discuss how Ripple Labs responds to the SEC's lawsuit to maintain Ripple's superiority and what the company will do in the future.

Based on our comprehensive analysis from three perspectives, we can summarize the overall state that Ripple currently has and therefore conclude its future strength and chance to survive. Although the prices of different cryptocurrencies will affect each other, the virtual currency market still has huge potential, and the price of XRP is likely to increase in the future.

This paper is organized as follows: Section 2 presents the institutional background of the cross-border transactions; Section 3 presents the analysis of Ripple from multiple perspectives; Section 4 concludes the evaluation of Ripple's long-term competitiveness.

\section{BACKGROUND}

Ripple is an emerging model of Internet Finance in the field of global payment, and it is an internet protocol designed for cross-border payment. This kind of agreement makes it efficient and cheap for any currency to transfer money to any corner of the world. Ripple operation needs two core elements: XRP and gateway. XRP is the base currency of RippleNet. It circulates in the whole RippleNet and can be sent to any user with a Ripple account in real time. RippleNet introduces the concepts of "consensus mechanism" and "gateway", and realizes transaction verification and confirmation in a short time through its distributed ledger framework and voting of special nodes [4]. At the same time, the automatic exchange rate conversion function in the system is used to realize the real-time exchange between currencies. Ripple is developed by Ripple Labs, which shows obvious characteristics of decentralization.

\subsection{Faster}

Under the traditional cross-border payment mode, banks process payment transactions at the end of the day, which generally takes at least 24 hours. And the manual reconciliation between banks also needs a period. The XRP cross-border payment based on blockchain is close to "real-time", and the transaction can be completed in 8 seconds.

\subsection{Cheaper}

The traditional cross-border payment mode has four costs: financial operation cost, receiving cost, payment processing cost, and settlement cost. The remittance fee is about $7.68 \%$ of the remittance amount. The average cost of cross-border payment between bank and agent bank is between $\$ 25$ and $\$ 35$, which is more than 10 times the cost of the domestic settlement with the automatic exchange center. With Ripple technology, banks can save $60 \%$ of the total processing cost. On average, when an enterprise pays $\$ 500$, the cost of payment processing will be reduced to $81 \%$. The cost of financial operation will be reduced to $23 \%$, and the cost of reconciliation will be reduced to $60 \%$.

\subsection{More liquidity}

In traditional cross-border payment mode, banks will hold multinational currencies in their bank accounts to maintain liquidity. As it is difficult for the collecting bank to predict when the agent bank will confirm the transfer information, the collecting bank must hold a certain amount of foreign currency in the account. In the Ripple cross-border payment mode based on blockchain, banks only need to use digital currency.

\subsection{More inclusive}

This is probably a revolution in trust. Choosing ripple means trusting blockchain technology. The mainstream way of international payment is SWIFT. Before Ripple, SWIFT established a unified way of account expression. In the original SWIFT network, only members can carry out cross-border payment transactions. This means that in the traditional cross-border payment mode, not all banks can join 
SWIFT. But Ripple payment mode is more inclusive. All banks, no matter big or small, are equal transaction subjects. The realization of democracy depends on the trust of all institutions using blockchain technology. The advantages of Ripple, such as real-time, near zero cost, and wider coverage, may have a serious impact on SWIFT [5].

\section{XRP'S COMPETITIVENESS FROM THREE PERSPECTIVES}

\subsection{Promising price tendency}

Among the famous cryptocurrencies, XRP is known for its reasonable price for both investment and cross-border transactions. XRP has low remittance fees compared to bank charges and provides more flexible and convenient ways for currency exchange. More people begin their cryptocurrency investment in recent years, especially when they see the profit that Bitcoin brings. The difficulty of getting Bitcoin when it only has a limited number in the whole world takes miners a lot of effort and usually high costs for people to invest in Bitcoin. At the same time, XRP is relatively easy to get and incurs less cost [6]. Figure 1 shows the price fluctuation of XRP since it was created. We can see that before mid-2017, the price was close to zero dollars. In 2018, the price rose to the highest, and then due to CoinMarketCap adjusting its algorithm, the price of XRP began to fall, and finally, it stabilized at about 0.5 to 0.6 dollars. Although XRP experienced a sharp rise and fall in 2018 and the price reached several times higher and then lower than before, the overall price ranged from $\$ 0.5$ to $\$ 3.2$ dollars. After that, the XRP always maintains a stable level, and the price is about $\$ 1$ dollar and hasn't changed too much after that. From this aspect, we can see that the price of XRP is not that high compared to the other popular cryptocurrencies such as Bitcoin, Ethereum, and the fluctuation is not that fierce when considering the investment. Therefore, we've seen an overall steady state of XRP that is mature enough for people to invest. Many treat XRP as an underlying asset to hold for future investment and even cross-border transactions, and it's completely reasonable when considering the stable price and potential value in the future.

To talk about the future potential value of XRP, we wouldn't worry so much about the decline of XRP price because it has a relatively stable price level even when there is a price shock (that is similar to 2018's price shock). At the same time, we take a positive attitude toward XRP's future value increase after comparing it with the other cryptocurrencies. The impulse response function shows that in the long run, cryptocurrencies such as Ethereum, and XRP, and Litecoin respond positively to the Bitcoin price shock [7]. Although it's quite impossible to accurately predict the future value of Bitcoin or any one of the cryptocurrencies, we are still confident about the whole market situation currently. Nowadays, the cryptocurrency market still has huge potential for growth as governments and corporations worldwide observed that the decentralized nature and blockchain technology could bring benefits. Therefore a lot of research is being conducted for regulated global usage [8]. From this perspective, as the cryptocurrency still has room to grow, we should expect the future growth of many cryptocurrencies, including the XRP.

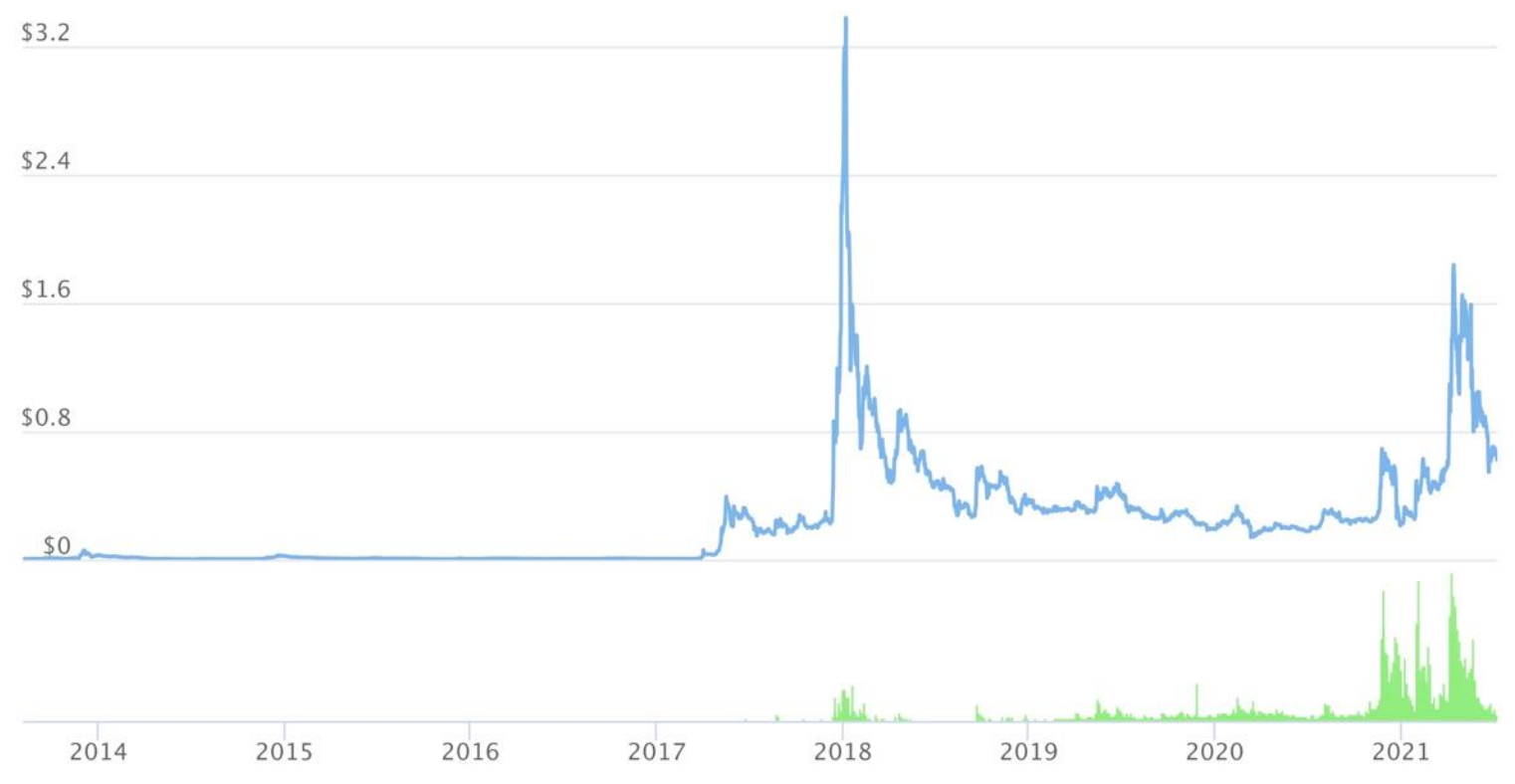

Figure 1 The time series of RXP price 


\subsection{Security and privacy}

Ripple's transaction method is largely based on its RippleNet, the major system playing a major role in taking frictionless cross-border transactions using blockchain technology. Blockchain technology appears as a new way of record-keeping, which is quite different from traditional methods of storing data in a central computer server. This technology allows multiple users to exist at the same time, and each of them is called a node, and every node can permanently store a certain amount of data along with the blockchain [9]. Each node has its unique identifier that even if an individual node is damaged, it doesn't affect the other nodes. Because of the separation of data, blockchain also keeps the stored information safer than the traditional method, where most data is stored together. While most cryptocurrencies use blockchain technology, it surely plays a role in maintaining the high-level security of RippleNet and providing better global services among many commercial institutions. As the Ripple company reported in the third annual Blockchain in Payments Report, blockchain technology benefits so many businesses from four perspectives: improved data quality, increased data security, cost-saving, and business growth [10].

Besides the blockchain, the creation of RippleNet itself has contributed a lot to security protection. Although it's a controversial topic that the use of RippleNet interferes with the decentralized blockchain, the private network somehow provides better security protection. From the beginning of the XRP market, Ripple Inc. marketed XRP as a better choice of digital currency than Bitcoin because of its stable price and incredible speed. The reason that XPR gains such a speed is the acceptance of the centralized network. It provides convenient data processing without too much proof of work. While some may worry about the "centralized" network that gathers information controlled by an individual company, customers of Ripple express their positive attitude toward the company. The Chief Technology Officer of Santander, one of the world's largest banks, indicates Ripple helps them "directly address the issue of speed and transparency around international payments" [11] by allowing them to know exactly the details of transactions and receipts.

While some people may be concerned why the speed of transactions contributes to safety and privacy protection, it's because this solves what people worry about: "electronic transfers of money are subject to double spending problems" [12]. The electronic transaction relies on digital files that play a role in transferring the value of money. However, the payee may be concerned that during the long period of waiting for the process, the same file has been sent to other payees that eventually makes the value of money decline. By removing the intermediate process of online money transactions that takes a lot of time and risk, Ripple further ensures customer information protection. In other words, Ripple is a global real-time gross settlement system (RTGS) that deals with frictionless cross-border payments via RippleNet.

In 2021, Ripple just got their SOC 2 (system and organization Controls 2) certification, a set of reports carried out by an independent CPA to certify the excellence in security and privacy. Together with the RippleNet Cloud, the Ripple system plays a good role in effectively and safely delivering the online transaction service. The Ripple system and XRP would therefore get high-level security that supports the further operation.

\subsection{Strong Support from Ripple Labs Inc.}

Distinct from some cryptocurrencies, XRP is the main product of Ripple Labs Inc., a company with a high level of competitiveness in the money transaction market. Ripple Labs is an operation that mainly focuses on cross-board real-time payment. To make their business more competitive, Ripple Labs introduces a brand-new idea -- the Ripple protocol. By using the blockchain concept, this idea does not depend much on the traditional concept, which is related to centralization. As a result, this new payment system benefits people and other companies from high efficiency, low cost, and so on [13]. To process the transactions, Ripple Labs developed a new cryptocurrency, XRP, which is widely used in RippleNet. With the creation of XRP, people can convert any type of currency into XRP and, at the same time, can convert XRP into any other currency. Compared to other cryptocurrencies, XRP can provide more convenience in the network from the transaction point. Taking the most well-known digital currency, Bitcoin, as an example, XRP is more efficient in transaction speed, transaction fee, privacy, and many other aspects. However, unlike many cryptocurrencies which use the idea of decentralization, even though the Ripple protocol, as mentioned before, applied this concept, XRP is not totally decentralized. On the contrary, it still needs deployment from the company. Thus the paper believes that Ripple Labs totally controls the XRP [14]. Because RippleNet depends much on XRP, the XRP will coexist with Ripple Labs. In this way, as long as Ripple Labs is widely used for its cross-board real-time payment business, the XRP market will always work as a medium for RippleNet. Moreover, Ripple Labs even wants to push the XRP to a larger platform in this day and age and play a greater role. In 2021, the Global Banking News claims that "In a white paper, Ripple has opined that its cryptocurrency, XRP, would have a role in central bank-issued digital 
currencies. XRP could become a 'neutral bridge' between different currencies" [15]. In this regard, we can see that Ripple Labs keeps using XRP in its own network and puts its efforts into making XRP more useful around the world.

From the company perspective, due to the support of the Ripple protocol, Ripple Labs has become much more popular in this field. After viewing the website of the Ripple Labs, there are hundreds of world-famous financial institutions, like Bank of America, Santander. They have joined the Ripple network and sought cooperation with it. By looking at its funding situation, we can see that Ripple Labs totally held five rounds of funding. The most recent one is Series C, conducted by Tetragon, a closed-ended investment company, and raised about 200 million at the end [16]. Moreover, many investors, such as SBI Holding, IDG Capital Partners, and so on, participated and invested in Ripple several times. The reason that Ripple Labs can attract that many investors and raise so much capital can be attributed to their advanced techniques. As a result, we consider Ripple Labs to be a strong company in this field, and with the help of Ripple Labs, XRP is able to exist in the long-term market and have a bright future.

\section{CONCLUSION}

Based on the analysis above, we can see that XRP as a cryptocurrency has the benefits of its stabilized price. In recent years, people worldwide pay more attention to the cryptocurrency market and are willing to make some investments in it. Compared to other digital currencies, we discovered that since XRP was created, the price did not fluctuate a lot. The price tends to stick to a price range from 0.5 dollars to 0.6 dollars in the long term. Because the price of different cryptocurrencies influences each other and the virtual currency market has great potential, the price of the XRP can grow in the future. From the aspect of security and privacy, XRP relies on its special system -- RippleNet, which applies the technique of blockchain. By utilizing the blockchain, people's information will be stored in the node and will not be affected by the others. This decentralized idea can offer people more transparent and faster trade. As of 2021, XRP got the system and organization Controls 2 certification, reflecting that XRP has a great advantage in privacy and security. Therefore, XRP's future circulation and transactions are guaranteed. In the last part, we analyze Ripple Labs, the company that created XRP. After searching its funding situation, we find that in the total five rounds of funding, especially the latest one, Ripple Labs raised more than 200 million dollars. Several companies participated in more than one round among those investors, which shows that investors are confident in this company. Ripple Labs applies the idea of blockchain, Ripple protocol, and RippleNet, in their cross-border transaction business, attracting many famous institutions to engage in it. However, unlike those decentralized products, the digital currency -XRP -- they created is totally controlled by Ripple Labs. As Ripple Labs is a powerful company in the money transaction market, XRP will coexist with Ripple Labs in the future. Moreover, the company intends to push $\mathrm{XRP}$ to the wider market, allowing XRP to develop in the future.

In conclusion, from value, security, and backup power aspects, we consider that Ripple will have a kind of bright future in the long term. However, some aspects still exist that people need to pay attention to in the future, such as the tightening policy from different countries, the new technology that can replace the blockchain, and so on, and adjust their decisions in time.

\section{REFERENCES}

[1] Deng Qing. Application Research of cross-border payment based on blockchain. 2020. Jiangxi Normal University, MA thesis

[2] Xu Jiayang. "Research on innovation of cross border payment system based on blockchain technology." financial education research 030.006 (2017): 9-14

[3] Youssef, Franck, and Erik Zenner. "Ripple: Overview and Outlook." Trust and Trustworthy Computing: 8th International Conference, TRUST 2015, Heraklion, Greece, August 24-26, 2015, Proceedings. Vol. 9229. Springer, 2015.

[4] Zhang Wei, and Chen Guihua. "Ripple mechanism structure, operation mode and regulatory thinking." financial accounting.06 (2017): 49-52. Doi: CNKI: Sun: jrkj. 0.2017-06-008.

[5] Liao Xiaoqiong, et al. "An analysis of the dynamic characteristics of the value of digital currency -Taking Bitcoin, ethereal currency and reborn currency as examples." finance and economy 2 (2021): 11-18.

[6] Reiff, Nathan. "What's the Difference Between Bitcoin and Ripple?" Investopedia, Investopedia, 7 July 2021, www.investopedia.com/tech/whats-difference-betw een-bitcoin-and-ripple/.

[7] Shen, Ze, David Bessler, and David Leatham. "How Cryptocurrencies Prices Affect Each Other?." (2019).

[8] Radovanov, Boris, et al. "A Time Series Analysis of Four Major Cryptocurrencies." FACTA UNIVERSITATIS - Economics and Organization, vol. 15, no. 3, University of Niš, 2018, pp. 271-78, doi:10.22190/FUEO1803271R.

[9] Brotsis, Sotirios, et al. "On the Suitability of 
Blockchain Platforms for IoT, Applications: Architectures, Security, Privacy, and Performance." Computer Networks, vol. 191, 2021, p. 108005., doi:10.1016/j.comnet.2021.108005.

[10] "Blockchain in Payments Report." Ripple, 15 Oct. 2020 , ripple.com/lp/blockchain-in-payments-report/?utm _content=header.

[11] "Ripple Customer Case Study: Santander." Ripple, 23 June 2021,

Ripple.com/customer-case-study/santander/.

[12] Nath, G. V. "Cryptocurrency and Privacy:

Economic Analysis of Law." SSRN

Electronic Journal, 2020, doi: $10.2139 /$ ssrn.3661287.

[13] Rosner, Marcel T, and Kang, Andrew. "Understanding and Regulating Twenty-First Century Payment Systems: The Ripple Case Study." Michigan Law Review, vol. 114, no. 4, Michigan Law Review Association, 2016, pp. 64981.

[14] Jani, Shailak. "An Overview of Ripple Technology \& its Comparison with Bitcoin Technology." (2018).

[15] "Ripple claims XRP has role in central bank digital currency." Global Banking News, 25 Mar. 2021, p. NA. Gale Academic OneFile, link.gale.com/apps/doc/A656185077/AONE?u=um user $\&$ sid $=$ summon $\&$ xid $=05454223$. Accessed 24 June 2021.

[16] Ripple, Team. "Ripple Caps Record Year With \$200 Million Series C Funding." Ripple, 16 Jan. 2020,

Ripple.com/insights/Ripple-caps-record-year-with200-million-series-c-funding/. 